\title{
Cardiovascular implanted electronic devices in people towards the end of life, during cardiopulmonary resuscitation and after death: guidance from the Resuscitation Council (UK), British Cardiovascular Society and National Council for Palliative Care
}

\author{
David Pitcher, ${ }_{1}^{1}$ Jasmeet Soar ${ }^{2}$ Karen Hogg, ${ }^{3}$ Nicholas Linker, ${ }^{4}$ Simon Chapman, ${ }^{5}$ \\ James M Beattie, ${ }^{6}$ Sue Jones, ${ }^{7}$ Robert George, ${ }^{8}$ Janet McComb, ${ }_{1}^{9}$ James Glancy, ${ }_{1}^{10}$ \\ Gordon Patterson, ${ }^{11}$ Sheila Turner, ${ }^{12}$ Susan Hampshire, ${ }^{13}$ Andrew Lockey, ${ }^{14}$ \\ Tracey Baker, ${ }^{15}$ Sarah Mitchell, ${ }^{16}$ the CIED Working Group
}

- Additional material is published online only. To view please visit the journal online (http://dx.doi.org/10.1136/ heartjnl-2016-309721).

For numbered affiliations see end of article.

\section{Correspondence to} Dr David Pitcher, Resuscitation Council (UK), 5th Floor, Tavistock House North, Tavistock Square, London WC1H 9HR, UK david.pitcher@resus.org.uk

First released: March 2015. Minor revision for print version: February 2016. Print version funded by the Resuscitation Council (UK) and British Cardiovascular Society. Review date: March 2020.

Received 10 April 2016 Accepted 12 April 2016
CrossMark

To cite: Pitcher D, Soar J, Hogg $\mathrm{K}$, et al. Heart 2016;102:A1-A17.

\section{ABSTRACT}

The Resuscitation Council (UK), the British

Cardiovascular Society (including the British Heart

Rhythm Society and the British Society for Heart Failure) and the National Council for Palliative Care recognise the importance of providing clear and consistent guidance on management of cardiovascular implanted electronic devices (CIEDs) towards the end of life, during cardiorespiratory arrest and after death. This document has been developed to provide guidance for the full range of healthcare professionals who may encounter people with CIEDs in the situations described and for healthcare managers and commissioners. The authors recognise that some patients and people close to patients may also wish to refer to this document. It is intended as an initial step to help to ensure that people who have CIEDs, or are considering implantation of one, receive explanation of and understand the practical implications and decisions that this entails; to promote a good standard of care and service provision for people in the UK with CIEDs in the circumstances described; to offer relevant ethical and legal guidance on this topic; to offer guidance on the delivery of services in relation to deactivation of CIEDs where appropriate; to offer guidance on whether any special measures are needed when a person with a CIED receives cardiopulmonary resuscitation; and to offer guidance on the actions needed when a person with a CIED dies.

\section{SUMMARY STATEMENT: MAIN MESSAGES}

This section highlights the main messages. Readers are advised to refer to relevant sections of the full text in order to ensure that these summary points are interpreted and used in context.

\subsection{Aspects of routine device management}

- Cardiovascular implanted electronic devices (CIEDs) include permanent pacemakers and implantable cardioverter defibrillators (ICDs). They provide effective treatment for many people by reducing symptoms and/or by preventing sudden cardiac death.
- Where there may be a later need to consider deactivation (ie, in people considering an ICD, including a cardiac resynchronisation therapydefibrillator (CRT-D) device), this possibility and the reasons for it should usually be explained as part of informed consent to implantation (see section 7.4). At routine review appointments, people should have the opportunity to discuss concerns regarding any aspect of their device, including end-of-life decisions (see section 6.4).

- It is recommended that written consent for device implantation and elective replacement is worded so that the recipient surrenders ownership of the device in the event of removal for clinical reasons or after death. Otherwise, the device remains the property of the recipient or of their estate (see section 7.14).

- People with implanted devices should carry with them information about the nature of their device, how to obtain expert advice and, where appropriate, how to deal with an emergency (see section 18).

\subsection{Towards the end of life}

- People with ICDs, including CRT-D devices, who are approaching the end of their life should be given opportunities to discuss the option of deactivation of their device (see section 6). Individual assessment and discussion of the relative benefits and burdens of elective replacement of any device (for battery depletion) is especially important when people are approaching the end of life (see sections 10.7, 11.7 and 12.9).

- The majority of decisions about deactivation towards the end of life arise in people with ICDs, including CRT-D devices (see section 12.4). It is very rarely appropriate to consider pacemaker deactivation as part of end-of-life care unless this is requested specifically by the patient (see section 10.5).

- Decisions about deactivation of any device should be shared decisions, with full involvement of the person themselves and of the healthcare team caring for them, and must be based on careful 
assessment of a person's individual circumstances at the time (see section 7.2).

- When people lack capacity to share in decision-making, decisions must be made in their best interests, must be made according to the law in that jurisdiction and must involve those with legal power to make decisions on behalf of the person. The views of those close to the patient should be considered when making a best-interests decision in such circumstances (see section 7).

- It must not be assumed that having a do-not-attempt-CPR (DNACPR) decision or being identified as dying automatically warrants ICD deactivation or that ICD deactivation automatically warrants a DNACPR decision (see section 7.13).

- The appropriateness of deactivation and the appropriate timing of deactivation differ with different devices. When considering deactivation, it is essential to understand the nature and purpose of the device in each individual person and to involve those responsible for management of the device (see Section B: paragraph 3).

- Effective and consistent communication with the person with the device, with those close to them, and with all members of the healthcare team is crucial to avoid misunderstanding and to enable good decision-making. Sensitive discussions about device deactivation should be undertaken by professionals competent in such communication. Discussions and decisions about device deactivation, including those at the time of consent to implantation, should be documented fully (see section 7.15).

- Discussion of deactivation of an ICD as part of end-of-life care should allow ample time for explanation, for an agreed, shared decision and for planned deactivation by a cardiac devices physiologist in the majority of cases. Use of a magnet to deactivate an ICD may be useful in an emergency setting, after discussion and careful consideration of its consequences (see sections 12.4-12.6).

- Healthcare provider organisations should have comprehensive policies governing device management, including deactivation of devices, to ensure that people with devices have prompt access to appropriate care and support, including access to emergency deactivation if required (see section 17).

- Device services should have a clear policy governing safe disposal of devices. If devices are retained by patients (or after death by their estate), they should be given clear information about potential hazards and how to avoid them (see section 16.7).

\subsection{During and after cardiopulmonary resuscitation (see section 15)}

- No special precautions are necessary when delivering chest compressions and/or ventilation in the presence of an implanted electronic device. When possible, wearing clinical examination gloves is recommended during any delivery of cardiopulmonary resuscitation (CPR) as a standard part of personal protection against infection.

- When a person with an ICD suffers cardiac arrest in a shockable rhythm, the device is expected to deliver a sequence of shocks to attempt to terminate the arrhythmia. If the device does not deliver such shocks or if the shockable rhythm persists, external defibrillation should be attempted.

- External defibrillator electrodes should not be placed over or close to implanted electronic devices.

- If a person with a pacemaker or ICD has return of spontaneous circulation (ROSC) after receiving CPR, the device should be interrogated and checked (usually by a cardiac devices physiologist) at the earliest opportunity.

\subsection{After death (see section 16)}

- If a person with a CIED suffers unexpected or sudden death, interrogation of the device should be considered to obtain information about cardiac rhythm and device behaviour immediately beforehand. This may help to establish the mechanism and cause of death.

- ICDs, including CRT-D devices, must be deactivated before any attempt is made to remove them or to perform an autopsy, to avoid risk of a shock to the person carrying out that procedure.

- All implanted electronic devices must be removed before cremation as they may explode when heated to a high temperature. Device services should have arrangements in place to ensure safe disposal of devices after removal.

\section{Section A: General, ethical and legal aspects}

\section{INTRODUCTION}

The Resuscitation Council (UK) (RC (UK)), the British Cardiovascular Society (BCS) (including the British Heart Rhythm Society and the British Society for Heart Failure) and the National Council for Palliative Care (NCPC) recognise the importance of providing clear and consistent guidance on management of CIEDs towards the end of life, during cardiorespiratory arrest and after death. This document has been developed to provide guidance for the full range of healthcare professionals who may encounter people with CIEDs in the situations described below and for healthcare managers and commissioners. The authors recognise that some patients and people close to patients may also wish to refer to this document. It is intended as an initial step

- to help to ensure that people who have CIEDs, or are considering implantation of one, receive explanation of and understand the practical implications and decisions that this entails;

- to promote a good standard of care and service provision for people in the UK with CIEDs in the circumstances described;

- to offer relevant ethical and legal guidance on this topic;

- to offer guidance on the delivery of services in relation to deactivation of CIEDs where appropriate;

- to offer guidance on whether any special measures are needed when a person with a CIED receives CPR;

- to offer guidance on the actions needed when a person with a CIED dies.

There has been a progressive increase over more than 50 years in the number of electronic devices implanted. This started in 1958 with the first implanted pacemaker and has progressed to include other devices, implanted to reduce or prevent symptoms, to reduce the risk of death, to prevent death by treating cardiac arrest, to monitor the heart's rhythm or any combination of those objectives. These devices are referred to collectively as CIEDs.

The increasing use of CIEDs has provided considerable benefit but has also created new challenges for patients and those close to patients, and for healthcare personnel caring for them. Particular challenges may arise when people, despite the presence of their implanted device, approach or reach the end of life. This may be due to deterioration in their heart condition (most commonly heart failure) that cannot be reversed by additional treatment or to the development or progression of another terminal or long-term condition such as cancer or chronic lung disease or kidney failure. 
With increasing frequency, questions arise about possible deactivation of some of these devices as part of end-of-life care when the continued operation of some devices may be of more burden than benefit to people. ${ }^{1-4}$

With increasing frequency also, healthcare professionals caring for such people are faced with practical questions as to how devices can be deactivated and what arrangements are in place in their particular locality to provide the equipment and expert support needed to assist with the management of a device. Policies and information about device deactivation are available in some but not all localities.

Practical considerations also arise and may lead to uncertainty when someone with an implanted device suffers a cardiorespiratory arrest and CPR is started. Those involved in attempted resuscitation may not have detail of the implanted device, may not be familiar with the precise nature and purpose of the device, may be unsure whether they should modify how they deliver CPR or may be unsure whether the device presents a risk to the people providing CPR.

Yet further practical considerations concerning the need for device deactivation or removal arise when someone with an implanted cardiovascular device has died.

This joint document will not

- offer guidance on the selection of patients for implanted devices

- offer specific guidance on temporary deactivation of CIEDs for other reasons (eg, during a surgical operation)

- offer detailed guidance on decisions relating to CPR

- offer detailed guidance on the delivery of CPR.

on all of which topics detailed published guidance is available (see 'References').

The following sections will consider the general principles of deactivation of devices, together with the ethical and legal considerations that apply, and the general principles of good clinical practice, including communication and informed consent for implantation. The nature and purpose of each individual type of device will be described in separate sections, together with the specific actions that are relevant to management of each type of device towards the end of a person's life, during CPR and after death.

\section{METHODS}

This guidance was produced according to the RC (UK) Development Process Manual (2014). The subject was chosen by the Executive Committee of the RC (UK) as the RC (UK) had received several queries concerning management of CIEDs in people towards the end of life, during CPR and after death. There was no existing detailed guidance on this topic and discussions with the BCS and the NCPC showed that there was interest in developing guidance on this topic.

The guidance was developed by a Working Group, convened by the RC (UK) on behalf of the three primary author organisations: RC (UK), BCS and NCPC. Membership of the Working Group is listed in online supplementary appendix C.

A scope for the guidance was developed and posted for consultation via the websites of the three primary author organisations. Applications for registration as stakeholders were invited from organisations considered to have a potential interest in the project, and it was made clear that stakeholder registration from any other interested organisation would be welcomed. A list of registered stakeholders is presented in online supplementary appendix B. Comments on the scope were received and considered by the Working Group when finalising the content and wording of the scope. Although the initial scope included consideration of ventricular assist devices, during development of the guidance the Working Group decided that these would be better addressed in a separate document and that this guidance should consider only CIEDs.

Literature searches were carried out by JS and DP to identify relevant publications, and updated in August/September 2014 (see online supplementary appendix D). In addition, Working Group members and stakeholders identified other documents, including local policies, and patient information leaflets. There are no specific trials in this area. Searches identified observational studies, reviews, expert opinion and case studies. The available evidence to support any intervention was therefore of low or very low quality, with a high risk of bias. The recommendations are therefore based on expert opinion, balancing of benefits and harms, and the values and preferences of the Working Group and stakeholders. Specific recommendations about the management of CIEDs during CPR were taken from the RC (UK) Resuscitation Guidelines 2010 and remain consistent with the RC (UK) Resuscitation Guidelines 2015..$^{5}$ These were produced using a National Institute for Health and Care Excellence-accredited process.

The method used to arrive at recommendations was based on review and discussion of evidence by the Working Group until consensus was achieved. A process of informal consensus was used. Each member of the Working Group had opportunities to express their views and engage in constructive discussions at each stage of development. A draft of the document was made available to all registered stakeholders for a consultation period of 4 weeks and was also reviewed and commented on by the Patient Advisory Group of the RC (UK). Received comments were considered individually by the Working Group and used to develop the final wording of the document where appropriate. The document was checked by legal experts.

Organisational and financial barriers to implementation were discussed by the Working Group and addressed in relevant sections if appropriate. To support implementation, a patient information leaflet on ICD deactivation towards the end of life and a clinical operational document on ICD deactivation towards the end of life have been developed and can be accessed from www. resus.org.uk or via online supplementary appendix A. The final version of this guidance was agreed by the Working Group. The above reference to Resuscitation Guidelines $2015^{5}$ was added following their publication, and online supplementary appendix A was edited in February 2016 to ensure that the resources listed remained accessible.

\section{PEOPLE (PATIENTS) CONSIDERED IN THIS DOCUMENT}

The ethical and legal elements of this document apply primarily to adults (aged $\geq 18$ years). These represent the large majority of people with the implanted cardiac devices described further in Section B.

The principles of decision-making and refusal of treatment by children and young people and of withdrawing or withholding treatment in a child are described in detail elsewhere. ${ }^{6} 7$ The legal principles are encapsulated in the Children Act 1989. This states that the child's welfare is paramount and, wherever possible, specific regard should be paid to the ascertainable wishes and feelings of the child. Once children reach the age of 16 , they are presumed in law to be competent to give consent for themselves for their own medical and social care and any associated procedures, including end-of-life issues. In most respects, they should be treated as adults-thus, if a signature is necessary 
on a consent form, they may sign for themselves. ${ }^{8}$ However, unless the competent child refuses to consent to such disclosure, it is good practice for competent children to be encouraged to involve their families in decision-making. The ethical and legal situation for children under the age of 16 years is more complex. In this situation, it is advisable to ensure involvement of a paediatrician with experience in end-of-life care and decision-making.

Most other elements of this document apply as much to children as to adults.

\section{DEVICES CONSIDERED IN THIS DOCUMENT}

This guidance refers to people with the following CIEDs:

- pacemakers-for treatment of bradycardia;

- biventricular pacemakers, also referred to as CRT-for treatment of heart failure-some biventricular pacemakers have only a pacemaker function (CRT-P) and some also have a defibrillator function (CRT-D);

- ICDs-for treatment of ventricular arrhythmia predisposing to sudden death-these include those CRT-D biventricular pacemakers that also have a defibrillator function;

- implantable cardiac event recorders (also known as implantable loop recorders or implantable cardiac monitors).

Brief reference will be made also to implantable neurostimulators.

In the remainder of this document, where reference is made to ICD deactivation, that refers to both deactivation of devices implanted primarily as an ICD and deactivation of the ICD function of a CRT-D device.

\section{CONSIDERATION OF DEACTIVATION OF DEVICES DURING LIFE}

\subsection{Maintaining surveillance of the balance of risk and benefit}

Any treatment prescribed or provided to a person will have the potential to cause burden or harm as well as to provide benefit. When a device is used as part of a person's treatment, it is important to maintain careful consideration of the relative risks and benefits of deactivation in that individual compared with the relative risks and benefits of leaving the device fully active. If the person has capacity, they must be involved in this decisionmaking process. If they do not have capacity, any decision must be made in their best interests (see sections 7.6, 7.8 and 7.9).

\subsection{Deactivation towards the end of a person's life}

For people with some types of CIED (ie, ICDs, including CRT-D devices-see section 12), consideration and discussion of deactivation should occur when it is recognised that they are entering or have entered the last few weeks or months of their life. This may be due to progression of their heart condition (usually heart failure) despite their device and all other relevant treatment, or may be due to the development or progression of another terminal condition. One important reason (but not the only reason) for considering deactivation of ICDs and CRT-D devices is to try to spare these people from receiving multiple shocks from their device as they are dying. Such shocks are relatively common during the last few hours or days of life. ${ }^{9}$ Failure to deactivate an ICD in a dying man in 2012 caused distress to his family and resulted in an out-of-court settlement by the National Health Service Trust. ${ }^{10}$

Care should be taken to ensure that people with heart failure have received appropriate specialist assessment and all relevant treatment for their heart failure before it is accepted that they need to consider end-of-life care. ${ }^{11-13}$ Confident recognition that people are approaching the end of life can be difficult in some conditions such as advanced heart failure, despite helpful guidance (eg, from the Dying Matters Coalition, the National Gold Standards Framework CIC and the Royal College of General Practitioners). ${ }^{14-16}$ Close collaboration among healthcare professionals, especially but not exclusively in general practice, cardiology and palliative care, can help to support patients in the presence of such uncertainty.

The appropriateness of device deactivation and the appropriate time to consider this will vary according to

- the informed person's wishes and views

- the person's individual clinical circumstances

- the type of device (see sections 10-14)

- the purpose of the device in each individual (sections 10-14)

- the likely burdens and harms of continued device operation

- the likely burdens and harms of deactivation.

Careful consideration of all these factors should be an integral part of care planning, intended to ensure that, whenever possible, as they approach the end of their life, people receive the care that they would wish to have in the environment of their choosing.

Decision charts to guide planned and emergency deactivation of ICDs as part of end-of-life care are included in online supplementary appendix A.

\subsection{Other reasons for deactivation or removal during life} Some people require temporary deactivation of a device when it is delivering treatment inappropriately or incorrectly, while measures are taken to achieve correct delivery of treatment. Temporary deactivation of an ICD may be necessary during certain interventions, such as surgery or radiotherapy. This document will not address these indications for deactivation; other guidance on these situations is available. ${ }^{17} 18$

\subsection{Documenting discussions about device deactivation}

Clear detail of what has been explained about device deactivation to patients and to those close to patients at the time of implantation should be documented in the health record. That documented information should be readily available to all healthcare professionals who may have to discuss these topics again during routine review visits or at a much later date when patients are approaching the end of their life. At routine review visits, patients should be given the opportunity to discuss any concerns or questions that they may have regarding any aspect of their device, including end-of-life decisions, but such discussion should not be forced upon people who have expressed a clear wish that they do not wish to have those discussions. Such expressed wishes should be documented in the health record.

\subsection{Requests by patients for device deactivation or removal}

In some situations, people may request deactivation or even removal of their implanted device, sometimes without understanding the full implications of their request. Any such request requires careful discussion and consideration of the reasons for the request and also explanation of the likely consequences, and whether it is technically possible to comply with the request. Even if the decision of an informed person with capacity seems unwise or illogical to a clinician or to the healthcare team, that does not mean that the decision should not be respected (see section 7.3 ). 


\section{LEGAL AND ETHICAL CONSIDERATIONS REGARDING DEVICE DEACTIVATION}

\subsection{The importance of individual assessment}

There is widespread misunderstanding on the part of people with devices, those close to them and many healthcare professionals over what will happen when a device is deactivated. This must be assessed and explained carefully on an individual basis. For example, in people with an ICD, deactivation of ability of the device to deliver a shock will have no effect on how they feel and will permit them to die naturally, without experiencing shocks from their device. Further detail of the effects of deactivation of each type of device is provided in sections 10-14.

\subsection{Making shared decisions about treatment}

Decisions about a person's treatment (including device implantation or deactivation) should be made jointly with any patient with capacity, following explanation of the balance of risks and benefits. ${ }^{19} 20$ As people approach the end of their lives, especially if this is the first time that deactivation has been raised, such discussions are sensitive and often difficult for patients, for those close to patients and for healthcare professionals. This is not a valid reason to avoid discussions about these important decisions.

\subsection{Deactivation is withdrawal of treatment}

Legislation on assisted dying is currently under consideration, but some people may be concerned that deactivation could be interpreted as such, and analogous to voluntary euthanasia or assisted suicide. That is not the case. Voluntary euthanasia and assisted suicide each involve an active intervention that in itself causes the person's death. The courts have confirmed that, when death follows withdrawal of treatment, the person's underlying condition is deemed the cause of death. Such withdrawal will be lawful, provided that it follows from the person's competent refusal of treatment or, alternatively, is in his or her best interests. In such situations, the healthcare professionals are released from any duty to provide treatment. Parallels may be drawn with withdrawal of other treatments, such as drug therapy, renal dialysis or artificial ventilation, the main difference being that CIEDs are implanted within a person's body. ${ }^{21}{ }^{22}$ However, implantation is not a basis to see deactivation as morally distinct from withdrawal of any other treatment.

If a person with capacity requests withdrawal of treatment, despite being fully informed of the likely consequences, healthcare professionals must comply with that request, even when they consider the request unwise or illogical or when the withdrawal of treatment is contrary to medical advice,. However, should an individual healthcare professional be unwilling to take action where there is a properly established decision to deactivate a device, it will be necessary to identify another healthcare professional to carry out deactivation. The General Medical Council and British Medical Association have each published guidance on withholding and withdrawal of treatment. ${ }^{20} 23$

Healthcare professionals who undertake clinical work outside the UK should note that laws relating to deactivation of devices differ in some countries. Clinical decisions must comply with the laws of the local jurisdiction.

\subsection{Informed consent at the time of implantation (or replacement)}

When their views were explored, most people with an ICD believed that it is important to inform patients about the possibility of later deactivation of their device, ${ }^{24}$ but many ICD recipients do not consider this, are not given information about it and have misconceptions about the role of their devices. ${ }^{25-28}$

The possibility of a later need to deactivate an ICD and the reasons for doing so should usually be explained as part of informed consent prior to implantation in anyone considering an ICD or CRT-D device. ${ }^{29}{ }^{30}$ Obtaining consent from a person for treatment requires provision to that person of sufficient, intelligible information to allow them to make an informed choice. $^{31}$ The information provided to support the process of informed consent should include explanation

- of the balance of benefits and harms or burdens of device implantation at the time;

- of how the balance of benefits and risks may change in the future;

- that a time may come when it is best that the treatment (specifically ICD shocks) stops;

- of what ICD deactivation involves, should it be considered in the future.

Provision of such information requires sensitive discussion with patients and, with due regard to confidentiality, those close to them. Healthcare professionals may find discussions about deactivation and end-of-life decisions easier in some settings than in others and easier with some people than with others. The discussion required with, for example, an elderly person with heart failure being offered an ICD will be different from and may be perceived by some as easier than the discussion required with a young person being offered an ICD as primary prevention for an inherited cardiac condition that has caused them no symptoms. While the information provided and the way in which it is explained should be tailored to the needs and circumstances of each individual, relevant explanation and provision of information should not be withheld from people simply because the healthcare professional perceives that discussion as difficult or considers that the extent and content of information is not yet directly applicable. In exceptional circumstances, the clinician seeking consent may consider that providing information and explanation about future deactivation may cause harm, in which case the withholding of information and the reason for it should be documented carefully. Failure to provide such information (without good reason) may be considered unethical and unprofessional, and may generate a significant problem for the person themselves and for those responsible for the person's care in the future. Failure to provide such information may also be unlawful and might be deemed to be negligent or a violation of the individual's human rights. ${ }^{19}$

\subsection{People who refuse information or discussion}

There may be some people who express a clear wish not to receive some or all of the information offered or not to engage in discussion about future decisions and the risks or burdens of treatment. ${ }^{32}$ Should that happen, the person's wishes should be respected, and details of the discussions and the patient's expressed choices in this regard should be documented in their health record.

\subsection{Implantation and deactivation of devices in people who lack capacity}

If a decision about provision or withdrawal of treatment is being considered in a person who does not have capacity, the decision must be made in the person's best interests. This will require consideration of the person's medical interests, plus his or her wider (social, cultural, religious or family) interests. In 
most situations, subject to confidentiality, those close to the patient should be consulted when determining the patient's best interests. In some situations, there will be a legal requirement to consult those close to the patient or the patient's nominated representative (eg, in England and Wales, a person who has been given a Lasting Power of Attorney ${ }^{33}$ to make decisions of this nature on behalf of the patient). The laws that define the actions required and people who must be consulted in that situation differ among the four nations of the UK:

- in England and Wales, the Mental Capacity Act 2005; 3334

- in Scotland, the Adults with Incapacity (Scotland) Act $2000 ;^{35} 36$

- in Northern Ireland, there is currently no specific statutory provision for decision-making for patients who lack capacity. A Mental Capacity Bill has been passed but has not yet come into force.

All healthcare professionals have a duty to be aware of and act within the laws that apply in their place of work.

\subsection{Explanation to patients who regain capacity}

Should a decision ever be made to implant a device in a person's best interests, when they do not have capacity, if they subsequently regain capacity, it is important that they are offered full information about their device and its benefits and potential burdens, as they would have been before device implantation had they had capacity. In the rare event that they then request deactivation, their request must be respected.

Some people may have sufficient capacity to consent to treatment but may not remember what was discussed. There should be ongoing provision of information to patients and to those close to patients in these circumstances. That information, including guidance contained in information leaflets, should contain clear explanation of the possible future need to consider device deactivation.

\subsection{The role of a welfare attorney}

In England and Wales and in Scotland, the laws provide for people to appoint a welfare attorney to make decisions on their behalf about medical treatment in the event of them losing capacity to make such decisions. Where a person has a welfare attorney with such powers, the welfare attorney must be involved in making any decision about treatment choices, including choices relating to withdrawal of treatment, and are under a duty to make decisions in the patient's best interests. In England and Wales, a personal welfare Lasting Power of Attorney authorises the attorney to give or refuse consent to the carrying out or continuation of life-sustaining treatment only if the document contains express provision to that effect. ${ }^{37}$

\subsection{Making a best-interests decision for a person without capacity}

Wherever possible, a person who lacks capacity to make a decision should still be involved in the decision-making process. Even if the person lacks capacity to make the decision, they may have views on matters affecting the decision and on what outcome they would prefer. Their involvement can help those making the decision to work out what would be in the person's best interests.

Whether or not there is a legally appointed welfare attorney or guardian with powers to make decisions about medical treatment on behalf of a person the above laws require the views of those close to the person to be taken into account when making a best-interests decision. The decision maker must also take into account any evidence regarding the person's previously expressed wishes or beliefs and values, so that a best-interests decision is based as far as possible on what the person would have decided or chosen had they had capacity. The views of those close to the patient about what the person's best interests are must be considered also. The laws regarding such best-interests decisions apply equally to provision of treatment and to non-provision or withdrawal of treatment. In Northern Ireland, there is currently no such statutory requirement but seeking the views of those close to patients would be regarded as best practice.

\subsection{Advance care planning towards the end of life}

When people with implanted cardiovascular devices enter the last few weeks or months of their life, the relative risks and benefits of continued treatment from the device should be kept under continuing review in the context of the altered priorities and wishes that patients have in these circumstances. Advance care planning ${ }^{38}$ with such people should include consideration of their wishes about both device deactivation and CPR attempts (see below). In England and Wales, the Mental Capacity $\mathrm{Act}^{33}{ }^{34}$ provides for people with capacity to make a formal advance decision to refuse treatment (ADRT) in the event that they subsequently lose capacity. In order to be valid, an ADRT that refuses life-sustaining treatment must be in writing, be signed and witnessed, and state clearly that the decision applies even if life is at risk. Such an advance decision might include (for example) a decision to refuse continued defibrillatory shocks from an ICD in defined circumstances (see section 'Implantable cardioverter defibrillators'). Such an ADRT would be legally binding in those defined circumstances.

\subsection{Pacemaker checks and elective replacement towards the end of a person's life}

While it is hardly ever necessary or appropriate to consider deactivation of pacemakers implanted for bradycardia or biventricular pacemakers implanted for treatment of heart failure, some people may choose to stop attending for routine pacemaker checks because they consider the burden of hospital visits for such checks no longer worthwhile. The risk of failing to attend for routine pacemaker checks in these circumstances will usually be low but will vary from person to person, and it is important to ensure that patients are offered information relevant to their individual circumstances, allowing an informed decision. Good communication and teamwork from all the healthcare professionals involved with the patient is an essential component of good quality care. Some pacemakers can be checked and monitored remotely, without the need for visits to a hospital clinic. Not all pacemakers have this capability and not all pacemaker centres use this type of pacemaker. When a person with such a pacemaker enters the last few weeks or months of their life, it is essential that healthcare professionals work together and communicate effectively to ensure that decisions about the management of the pacemaker are not made purely on the basis of information from remote monitoring, without considering the patient's individual circumstances and wishes. For example (as discussed in section 10), arrangements for elective generator change for battery depletion should not be made by a pacemaker service solely on the basis of technical information, without regard to the patient's individual clinical circumstances and wishes. It is therefore crucial also that healthcare professionals in other settings and services, including general practitioners, contact pacemaker centres with relevant clinical information when a patient with an implanted electronic device is approaching the end of life. 


\subsection{ICD checks and elective replacement towards the end of a person's life}

If a person with an ICD enters the last few weeks or months of their life, re-evaluation and discussion of its benefit become appropriate for all but the minority of patients who do not wish to engage in such discussions. This is part of planning for their end-of-life care, and the healthcare professionals from all disciplines involved with that person should communicate effectively to contribute to this.

As with pacemakers, ICD batteries may become depleted coincidentally in a person who is nearing the end of their life. Elective replacement of the device provides an opportunity to review the balance of benefits and burdens of continued ICD therapy. For some people in the last few weeks or months of life, the benefit of elective device replacement may still exceed the burden, but for others it may be better to avoid the burden of elective replacement. ${ }^{39}$ As with other decisions referred to in this guidance, each decision must be based on careful individual assessment. The views of the informed patient with capacity are a crucial determinant of the decision. Decisions for those who lack capacity must be made in their best interests.

Where ICD deactivation has been decided with the patient, elective ICD generator replacement for battery depletion is unnecessary unless the patient is dependent on the ICD for pacing (see sections 10, 11 and 12). Where there is agreement not to replace a device in the presence of battery depletion, its removal would not usually be recommended as the harms and burdens of removal would be likely to exceed any benefit.

\subsection{Decisions about ICD deactivation and decisions about CPR}

An important relationship to consider is that between decisions to deactivate an ICD and decisions about CPR attempts in the event of cardiorespiratory arrest. A default, without scrutiny, that having a DNACPR decision or being identified as dying automatically warrants ICD deactivation or that ICD deactivation automatically warrants a DNACPR decision in every person is unethical. All decisions must be based on careful assessment of each individual situation. ${ }^{40}$

In the majority of circumstances as a person nears the end of their life, if there is an agreed decision with a patient or their representative that ICD shocks would present more burden than benefit, the same decision will be made about CPR, given that it is more traumatic and invasive, with less likelihood of a successful outcome. If it has not occurred already, a DNACPR decision should be discussed at the same time as discussion of ICD deactivation, but recognising that there may be occasional situations in which the person will wish to be considered for CPR despite choosing to have their ICD deactivated.

Some healthcare professionals express a view that the converse should apply, namely that a DNACPR decision always implies that an ICD should be deactivated. For people with an ICD, a DNACPR decision or the recognition that they might be dying should trigger a discussion about ICD deactivation. However, situations may arise in which a fully informed person chooses not to have CPR attempted because of its trauma or relatively low probability of success, but chooses to continue to receive treatment from their ICD for shockable ventricular arrhythmia. There may be situations in people who are nearing the end of life where an ICD is deactivated because it is delivering inappropriate shocks in the absence of ventricular arrhythmia, but the patient still wishes to receive CPR in the event of cardiac arrest. These choices must be respected and kept under review with the opportunity for decisions to be changed as the person's condition progresses.

\subsection{Ownership of implanted devices}

Disputes over ownership of implanted devices are very rare, but many clinicians are unfamiliar with the position regarding ownership of devices. There is no legislation covering the question of ownership of implanted medical devices, including internal cardiac defibrillators and pacemakers. As far as we are aware, there is currently no case law on this matter in the UK.

In 1983, the Department of Health and Social Security gave guidance in circular $\mathrm{HN}(83) 6^{41}$ on the ownership of various implants, including cardiac pacemakers, and the removal of cardiac pacemakers after death. Although it pre-dates the widespread use of CIEDs other than pacemakers, this guidance has not been revised. In 2011, the Department of Health issued a statement endorsing the 'default position' set out in $\mathrm{HN}(83) 6$ that the device is owned by the individual into whom it is implanted.

$\mathrm{HN}(83) 6$ states:

On implantation an implant becomes the property of the person in whom it has been implanted and it remains his or her property even if it is subsequently removed. Following the patient's death it forms part of his or her estate unless there is any specific provision to the contrary.

This wording may be misleading. The general position of the law is that neither the whole living body nor the whole deceased body are property. There can be property in parts separated from the living and the deceased in particular circumstances. While the law in this area is limited and not altogether clear, one of the main property law doctrines is that only things that are separate from persons (ie, the body) can be owned and subject to property rights. This doctrinal position has been affirmed by the courts in relation to biomaterials (ie, items or materials that originated within the body): to be the subject of property rights, they must be separate from the person/body. Biomaterials that have been separated from the body may, therefore, in some circumstances become subject to property rights. An external medical device may be classified as personal property. When a device is implanted, it becomes part of the living body and, in some cases, becomes integral to the very functioning of the recipient. However, it is difficult to say definitively what its 'property' status is once it becomes part of the body since this specific point has not been tested in the courts (in England at least). Interrogation of a device to obtain stored data (see sections 16.1-16.3) will be governed by regulations on data management and health records (Quigley $M$, personal communication).

The question of ownership may arise when an implanted device is removed from the body during life or after death. The notice $\mathrm{HN}(83) 6$ provided an agreed modified wording for consent forms for implants to try to avoid the possibility of dispute about the right of a healthcare provider organisation or consultant to retain an implant removed for examination or replacement. In the revised form of consent, the patient would sign an agreement stating:

I acknowledge and agree that any implant supplied to and implanted in me as part of this operation or the further or alternative operative measures referred to above, is supplied and implanted subject to the condition that if at any time it is removed by or on behalf of a health authority:

(a) for the purpose of replacement, or

(b) where a replacement is not required to enable it to be 
examined, or

(c) where in the case of a cardiac pacemaker paragraph (a) or (b) does not apply, after my death the ownership of the implant will vest in that health authority.

The 2011 statement indicated that in any specific case where such provisions are explicitly made, either on a preoperative consent form or subsequently, legal ownership may reside with a healthcare provider organisation or party other than the patient or their estate.

To minimise confusion, services and their responsible clinicians involved in device implantation will need to consider modification of the above wording on forms of consent

- so that it relates accurately to the type of device being offered, should that not be a pacemaker;

- so that ownership is vested in the healthcare provider organisation removing the device (which may have been implanted elsewhere).

In situations where the person's prior agreement has not been obtained, given the legal ambiguities, it is advisable to proceed as if the patient has ownership of the device. Therefore, their consent is required for retention of a device that is removed for clinical purposes during life, but also consent should be obtained from the executor(s) of their estate for removal of a device after death, and for retention and disposal of that device. As there may be practical difficulties in identifying and contacting executors, obtaining prior consent from the patient for removal, retention and disposal after death is recommended whenever possible. Should the patient, or after their death the executors or beneficiaries of their estate, choose to keep a device that has been removed, healthcare providers should offer them clear advice on any potential risks or hazards that could result (see section 16.7).

\subsection{Communicating and recording information}

Effective communication and documentation are essential components of good-quality clinical care. Failures of these elements of care are among the most common reasons for dissatisfaction, complaint and litigation.

Communication with patients and those close to them about ICD deactivation (including about possible deactivation in the future), as with all aspects of end-of-life care, requires complex, sensitive discussion. This should be undertaken by experienced members of the healthcare team with the competence and knowledge to undertake such conversations. Healthcare organisations have a responsibility to ensure appropriate training for staff who undertake these discussions (see section 8). Communication with patients and those close to patients must be tailored to individual needs. When ICD deactivation is considered, careful explanation of exactly what it involves is needed in all cases, and many people will need firm reassurance that deactivating their ICD will not cause their death and that they will feel no different following deactivation. It is important to ensure also that people understand that if their condition improves or they change their mind, their device can be reactivated.

All discussions and decisions about device deactivation (and all other aspects of end-of-life care) must be communicated effectively among all other members of the healthcare team involved in any person's care, including usually the GP, the cardiology team and the palliative care team, and often other disciplines.

All discussions and decisions about device deactivation must be documented fully. That recorded information must be readily available to those involved in the person's subsequent healthcare.
When a decision is made with a person that their ICD will be deactivated, this action will often be performed by another healthcare professional (usually a cardiac devices physiologist). It is essential that the decision, the reason for making it and the involvement of the patient and/or those close to them are documented fully, so that the person performing the deactivation has all the information needed to allow them to proceed, and that they then document fully the action taken. This is best achieved using a standard proforma, of which examples in current use are included in some of the policies listed in online supplementary appendix A.

\section{DISCUSSION OF DEACTIVATION WITH PATIENTS AND THOSE CLOSE TO THEM}

\subsection{Training and competence in communication skills}

Formulating individualised end-of-life care plans with or on behalf of patients is always a sensitive process and requires healthcare professionals to be competent in undertaking such discussions. Employers have a duty to ensure that professionals who are required to undertake such discussions are both trained and competent.

\subsection{The healthcare professionals who should be involved in the discussion}

The appropriate member of the healthcare team to have this conversation will vary. In the vast majority of cases in which deactivation of a device is considered during life, the consultant or senior clinician responsible for management of the patient's device should be involved in the decision-making process, but the degree of that involvement or its delegation will vary according to individual circumstances. Good communication within the entire healthcare team and with the patient and those close to them lies at the heart of the process so that there is clear and consistent information and advice and the decisions are agreed and understood by all.

Depending on individual circumstances, the healthcare professionals who initiate and undertake these discussions or provide support and information to patients and those close to them may include

- cardiologists

- heart failure specialist nurses

- arrhythmia specialist nurses

- cardiac physiologists (especially those involved in device management)

- general practitioners

- non-cardiologist physicians or surgeons

- palliative care doctors or specialist nurses.

The person who initiates a discussion will usually be a healthcare professional who is closely involved in the person's care and who knows them and their clinical and home circumstances. It may be necessary to involve several members of the healthcare team and to have serial discussions with patients and those close to them before reaching a shared decision that they are comfortable with.

\subsection{Multidisciplinary end-of-life care cardiology services}

Where available, a multidisciplinary end-of-life care service involving specialist healthcare professionals from cardiology and from palliative care may offer an environment that supports patients and those close to them in various aspects of their end-of-life care planning. The support needed should be considered on an individual basis and may also include help from other healthcare disciplines (eg, physiotherapy) spiritual advisers or from other agencies (eg, social workers). Where these specific services are not available, development of local clinical pathways 
can facilitate close multispecialty collaboration, in particular between cardiology and palliative care services.

\subsection{Aims of end-of-life-care planning in people with devices}

The objective should be to avoid a person entering their last few weeks or months of life, even acutely or unexpectedly, without a care plan or without their views about device deactivation being known. It is considered bad practice for the healthcare team that knows the person not to have anticipated such a situation and to have left this difficult task to, for example, a hospital acute admission team. Whenever a person with an implanted device presents with an acute clinical problem, early communication with and involvement of those usually responsible for the person's care and the management of their device should be routine.

\subsection{Discussions with those close to patients}

Involvement of those close to patients in discussions (with due regard for confidentiality) is important, both to provide support for the patient as they make decisions about their end-of-life care and to help their family and carers to understand how the person's 'health journey' is unfolding. While this can present challenges, reasonable effort must be made to engage them in the process. Seeking a single representative is one option, but can present problems if there is discordance within the family. All these interactions and processes should be documented clearly.

\subsection{Discussions with those close to patients who lack capacity}

Where the person has given legal authority to someone else to make decisions for them (eg, in England and Wales, under a Lasting Power of Attorney), that person must be involved in the decision-making process (see section 7.8).

In all other circumstances where the patient does not have capacity, the role of those close to them is to help healthcare professionals come to a best-interests decision by clarifying, as far as they are able, the patient's wishes, beliefs and values when they had capacity, as well as their own views as to what decisions are in the person's best interests. It is crucial that those close to patients understand clearly that they are not being asked to make a decision to deactivate a device that has been part of the person's treatment.

\section{INFORMATION THAT PEOPLE SHOULD RECEIVE 9.1 Guiding principles}

- Some understanding of the nature and purpose of a device is a prerequisite to informed decision-making about management of their device from implantation to death.

- Information-giving should be a priority from the time that device implantation is first considered, to give people every opportunity to understand its nature and purpose.

- Information provided must be clear and understandable.

- Good communication requires the professional to ensure that the information given has been understood correctly.

\subsection{Verbal communication}

As they approach the end of their life, most people will need several opportunities to discuss deactivation of their device and contribute meaningfully to a shared decision. While clear conversation is only one component of information-giving, the inclusion of a trusted friend or family member in a discussion may be of help to some people. Where conversations cross languages, an independent interpreter may be necessary.

\subsection{Written/printed information}

Written information is important and should be available in languages relevant to a locality, should be culturally sensitive and should signpost people to additional support or resources. Written information should never be regarded or used as a substitute for a clear, spoken explanation and the opportunities for patients and those close to patients to ask questions and have them answered.

The British Heart Foundation has developed a guide for healthcare professionals on deactivation of ICDs towards the end of life. ${ }^{42}$ Other helpful sources of information about the devices under consideration are available from national organisations and in individual healthcare regions and districts. To assist with implementation of this guidance, the Working Group has developed a clinical guide on deactivation of ICDs towards the end of life and, in collaboration with the British Heart Foundation and the Arrhythmia Alliance, an information leaflet for patients and carers on deactivating the shock function of an ICD towards the end of life (https://www.resus.org.uk/ defibrillators/cardiovascular-implanted-electronic-devices/).

\section{Section B: Device-specific aspects}

While many of the above aspects of ethics, the law and communication are generic to all types of implanted device, there are major differences in the nature and purpose of different types of device, and therefore major differences in the decisions and actions that may be needed as people approach the end of life, after death or in the event of cardiorespiratory arrest. In this section, information is included in particular to help nonclinicians and clinicians with no specialist knowledge of devices to understand the types of device, the differences in their nature and purpose, and the resulting differences in clinical decisionmaking that may be needed.

Information and guidance is provided also for all of the circumstances in which it may or may not be appropriate to consider deactivation or non-replacement of each type of device when people are approaching the end of life and on the procedures involved in deactivation. Furthermore, device-specific information is provided concerning any actions or precautions required by those attempting resuscitation when someone with an implanted device suffers cardiorespiratory arrest.

Healthcare professionals who are or who become involved in the care of patients with implanted devices need clear information about the specific nature and purpose of the device in each individual patient, and should make every effort to obtain such information to assist with decisions about treatment. Furthermore, if adjustment of a device is necessary, physiologists and other members of the specialist (usually cardiology) team will need such information, including details of the manufacturer and model of the device. Many people with implanted devices carry such information with them, but if necessary in urgent or emergency situations details should be obtained from the implanting centre, the centre providing ongoing surveillance of the device if that is not the implanting centre or the patient's general practitioner.

It is important to ensure safe disposal of any implantable electronic device after removal during life or after death. This aspect is discussed further in section 16.7.

\section{PACEMAKERS FOR BRADYCARDIA}

\subsection{Pacemakers for bradycardia: nature and purpose}

These pacemakers are implanted to prevent the heart from beating inappropriately slowly. They consist of a 'generator' (the 
pacemaker itself), often implanted under the skin in the pectoral region, and one or two insulated leads that connect the pacemaker to the heart. Single-chamber pacemakers have a single lead, connected either to a lower chamber (ventricle) of the heart, usually the right ventricle, or an upper chamber (atrium), usually the right atrium. The choice of connection to atrium or ventricle will be determined by the underlying condition for which the pacemaker was implanted. Dual-chamber pacemakers have two leads, one connected to an atrium and one to a ventricle.

\subsection{Pacemakers for bradycardia: reasons for implantation}

The majority of such pacemakers are implanted for the treatment of sinus node disease ('sick sinus syndrome') or atrioventricular (AV) conduction disease ('heart block'); less common indications include treatment of some forms of severe reflex syncope (such as vasovagal or carotid sinus syncope). In many people, the main purpose of the pacemaker is to prevent or reduce symptoms that result from bradycardia, such as syncope, sudden feelings of faintness ('presyncope') or fatigue and breathlessness. In some people (mainly those with advanced AV conduction disease), the pacemaker will also reduce a risk of dying suddenly.

\subsection{Pacemakers for bradycardia: non-invasive adjustment}

People with pacemakers are not aware of the tiny electrical impulses that the pacemaker uses to stimulate heartbeats. Adjustments to the way in which a pacemaker detects and responds to the heart's natural, spontaneous electrical signals can be made non-invasively without any discomfort using a programmer that communicates with the generator through the skin overlying the pacemaker.

\subsection{Pacemakers for bradycardia: pacemaker dependence}

Some people with a pacemaker for advanced AV conduction disease become 'pacemaker-dependent', meaning that no prompt spontaneous heartbeats occur if the pacemaker ceases to stimulate heartbeats. For these people, sudden 'switching off' of the pacemaker would be likely to lead to loss of consciousness (until a delayed spontaneous heartbeat occurs repeatedly) or death (if a spontaneous heartbeat does not occur or is not sustained).

\subsection{Pacemakers for bradycardia: need for deactivation is rare}

It is very rare for people who have pacemakers implanted for bradycardia to need deactivation of their devices during life. As many people with such pacemakers have them implanted to reduce symptoms, continued control of those symptoms remains an important part of their end-of-life care. Some people raise concern that the presence of a pacemaker may delay their death and in some cases prolong suffering, by preventing the heart from stopping, and it is important to explain to them that the pacemaker will not usually prevent or delay natural death as in many cases the final heart rhythm is a ventricular arrhythmia, which would not be prevented by the pacemaker. If a person who is pacing-dependent asks for their pacemaker to be 'switched off', it is important that they understand that doing so may lead to their immediate death but could also result in a distressing episode of syncope, during which they may suffer harm, and after which they may be left with continuing burdens such as a new disability or distressing symptoms.

\subsection{Pacemakers for bradycardia: method of deactivation}

In the exceptionally rare situation where the healthcare team and the patient and/or those close to the patient decide that deactivation of a pacemaker is in the person's best interests, this can be performed using a programmer, as described in section 'Pacemakers for bradycardia: non-invasive adjustment' above. Placing a magnet over the pacemaker will not deactivate its pacing function but will cause the pacemaker to fire at a faster than usual rate and will prevent the pacemaker's ability to be inhibited by spontaneous heartbeats. Magnets should not be used in a misguided attempt to deactivate such a pacemaker.

\subsection{Pacemakers for bradycardia: management of reduced battery life}

One dilemma that is not uncommon arises when it is found that a person who is approaching the end of their life has a pacemaker with reduced battery life, such that elective replacement of the generator (often referred to as a 'box change') would usually be advised. As with all clinical decisions, the decision whether or not to proceed with generator replacement must be determined by careful assessment of the individual circumstances in each person, and whenever possible patients should contribute to the decision after receiving the necessary information and explanation to allow them to do so. The relative burdens and benefits of elective replacement versus allowing the battery to run down will be influenced by various factors including

- the views and wishes of the patient

- the underlying indication for pacing

- whether or not the person has become pacing-dependent

- how soon the person is likely to die

- options to reprogramme the pacemaker to minimise further battery depletion.

Hospitals that provide pacemaker services should ensure that when a pacemaker is found to warrant consideration of generator replacement, the decision to proceed is made on the basis of proper informed consent (or in the person's best interests if he/she lacks capacity), in the full context of the individual person's current clinical circumstances. Automatic listing of patients for generator replacement based only on the state of the pacemaker battery is poor practice and should be avoided.

\section{BIVENTRICULAR PACEMAKERS}

\subsection{Biventricular pacemakers: nature and purpose}

These devices are implanted primarily to try to improve the mechanical pumping action of the heart. They will also provide effective treatment of bradycardia (as above) should this be required. When there is reduced contraction of the left ventricular myocardium, this leads to symptoms of the clinical syndromes that are referred to as 'heart failure'. First-line treatment for heart failure is with drug therapy, but for some people with troublesome symptoms from heart failure despite appropriate medication, using a pacemaker that stimulates the right and left ventricles virtually simultaneously produces a more coordinated contraction of the ventricles, resulting in a more effective pumping action and, for many but not all, substantial reduction in the symptoms of heart failure. ${ }^{43}$ Use of biventricular pacemakers for this purpose is also referred to as 'cardiac resynchronisation therapy'. Where the device has no capability other than this pacemaker therapy, it is referred to as CRT-P (see also section 'Biventricular pacemakers: some people also need an ICD').

As with pacemakers for bradycardia, the generator is usually implanted in the pectoral region. These devices usually have three leads, one connected to the right atrium and one connected to each of the ventricles, but in some people an atrial lead is not required.

For further detail, see Singh and Gras. ${ }^{44}$ 
11.2 Biventricular pacemakers: non-invasive adjustment

These pacemakers also use tiny electrical impulses to stimulate the heart and people with this type of pacemaker are not aware of these. Adjustments to the function of biventricular pacemakers can be made non-invasively without any discomfort, in the same way as with pacemakers used to treat bradycardia.

\subsection{Biventricular pacemakers: some people also need an ICD}

Some people requiring biventricular pacing are also at sufficient risk of sudden death to warrant use of an ICD (see below). Such people have a device capable of delivering defibrillation as well as cardiac resynchronisation (CRT-D). The two functions of these devices can be adjusted or deactivated independently from each other and the balance of benefits and burdens of each can therefore be considered separately in each individual person.

\subsection{Biventricular pacemakers: effect of deactivation or failure}

Unless the person is pacing-dependent (as described above), a person with a biventricular pacemaker would be unlikely to be aware of any immediate, severe symptoms should the pacemaker be switched off or suddenly cease to function, but cessation of biventricular pacing is likely to be followed by worsening of symptoms of heart failure in those people whose heart failure symptoms were reduced by this treatment.

\subsection{Biventricular pacemakers: deactivation and non-replacement}

As with pacemakers for bradycardia, it is rare for people to require deactivation of the pacemaker function of their devices. Deactivation of a biventricular pacemaker could lead to an increase in symptoms of heart failure and increase the distress of a person as they approach the end of their life. As with pacemakers for bradycardia, careful consideration and discussion of the relative risks and benefits of generator replacement will be needed on an individual basis should battery depletion develop, and there is no place for blanket policies based only on the state of the pacemaker batteries.

\subsection{Biventricular pacemakers: methods of deactivation}

In the very rare situation where the healthcare team and the patient and/or those close to the patient decide that deactivation of a CRT-P device is in the person's best interests, this can be performed using a programmer (usually by a cardiac devices physiologist), as described above. If a person has a CRT-P device, placing a magnet over the pacemaker will not deactivate its pacing function but will cause the pacemaker to fire at a faster than usual rate and will prevent the pacemaker's ability to be inhibited by spontaneous heartbeats. Magnets should not be used in a misguided attempt to deactivate a CRT-P device.

The ICD function of a CRT-D device can be deactivated using a programmer, without interrupting its pacemaker function. Application of a magnet will also suspend tachyarrhythmia detection and thereby suspend delivery of shocks (and of bursts of very rapid pacing to try to interrupt ventricular tachycardia (VT)) by the device and can be used to provide emergency, temporary deactivation of the defibrillator function of the device without interrupting its biventricular pacemaker function.

These distinctions underline the importance of healthcare professionals who are caring for a person having clear information about the nature and purpose of the implanted device in that individual person, and obtaining timely expert help from a cardiac devices physiologist and/or cardiologist in managing that device.

The factors to be considered in relation to deactivation of the defibrillator function of CRT-D devices are described further in section 12 .

\subsection{Biventricular pacemakers: management of reduced battery life}

Reduced battery life in a CRT-P device should be managed in the same way as for a pacemaker implanted for bradycardia (see section 10.7). Reduced battery life in a CRT-D device should be managed in the same way as for an ICD, with due regard to its pacing function (see section 12.9).

\section{IMPLANTABLE CARDIOVERTER DEFIBRILLATORS 12.1 ICDs: nature and purpose}

ICDs are implanted primarily to deliver a defibrillatory shock when the patient develops a ventricular arrhythmia that is an immediate threat to their life, such as cardiac arrest in ventricular fibrillation (VF). Many of these devices are programmed also to deliver rapid pacing stimuli that may interrupt a VT that, if it continues, carries a high risk of causing the person harm or of progressing to cardiac arrest. ICDs may be implanted on the basis of 'secondary prevention' in people who have already suffered one or more episodes of VF or VT, or may be implanted as 'primary prevention' in people who are at high risk of developing VF or life-threatening VT. In addition to the functions described, ICDs have a back-up pacemaker function that will stimulate heartbeats if the person develops bradycardia, in the same way as a pacemaker that is implanted purely to treat bradycardia. As discussed in section 11, some ICDs are implanted as CRT-D devices to deliver biventricular pacing for heart failure as well as to provide a defibrillator function because of a risk of ventricular arrhythmia causing sudden death.

In most patients, ICDs are implanted in a similar subcutaneous pectoral position to that used for pacemakers. The devices are larger than pacemakers. Most ICDs use transvenous leads in the same way as with pacemakers. A more recent development that may be suitable for some but not all ICD patients is the subcutaneous ICD. ${ }^{45}$ This has no transvenous leads. It can deliver a defibrillatory shock and has limited pacemaker capability. The generator of a subcutaneous ICD is usually implanted in the left lateral chest wall.

\subsection{ICDs: awareness of shock delivery}

Although people with ICDs are unaware of the low-energy stimuli from their device when it is acting as a pacemaker, the higher-energy shock needed to defibrillate life-threatening ventricular arrhythmia is unpleasant and painful. In some situations, the person will have lost consciousness and collapsed before the device delivers a shock, sparing them the discomfort of the sudden shock, but not the distress or risk resulting from the collapse (unless they lost consciousness while asleep). There are also circumstances in which ICD shocks are delivered to patients who are fully conscious, and in some people such shocks may be more likely to occur and may occur repeatedly in those who are approaching the end of life.

\subsection{ICDs: effect of deactivation or device failure}

If an ICD suddenly ceases to function completely, the patient will be unaware of this, unless they use the ICD also for its function as a pacemaker (see sections 10 and 11). However, if they subsequently develop VF or VT, they will suffer cardiac 
arrest (or symptoms from the arrhythmia in some cases of VT). If an ICD is deactivated, that involves switching off its tachyarrhythmia detection function, so that it will not deliver a shock or a burst of rapid pacing. Its back-up function as a pacemaker is not deactivated or otherwise affected, so the patient will not feel any different after deactivation.

\subsection{ICDs: decisions about deactivation}

The majority of decisions that are needed in relation to device deactivation arise in people with ICDs. The primary reason for having an ICD is to prevent sudden death, so when it is recognised and accepted by a person and their healthcare professionals (and where appropriate by people close to these patients) that the person is approaching the end of their life and that the focus of treatment has shifted to control of symptoms rather than attempts to prevent death, it is usually appropriate to consider and discuss deactivation of the defibrillator function of the ICD. The aim is to ensure that the person does not experience unpleasant shocks from the device that cause more distress than benefit. In some cases, such shocks may prevent a natural death and prevent a relatively peaceful and dignified release from distressing symptoms (eg, symptoms of heart failure).

\subsection{ICDs: method of planned deactivation}

Tachyarrhythmia detection by an ICD can be deactivated, suspending its defibrillator function without affecting its ability to function as a pacemaker, if it has a pacemaker function. ICDs can be reprogrammed or deactivated using a programmer that transmits signals to the device through the skin overlying the device. These programmers are the same as those used to test and reprogramme pacemakers and are usually operated by cardiac physiologists involved in delivering pacemaker and ICD services. Current programmers are specific to the manufacturer of the device, emphasising the importance of providing a physiologist with details of the individual device whenever possible. These programmers and their operators are usually based in hospital Cardiology Departments, so for most people reprogramming or deactivation of their ICD requires them to attend their local pacemaker/ICD department. However, the programmers can be transported, so there may be local arrangements that would allow a cardiac devices physiologist to visit a patient in another healthcare facility or in their home to deactivate an ICD as part of their end-of-life care. In the majority of people approaching the end of life, consideration and planning of ICD deactivation should take place in advance, allowing deactivation using a programmer to be performed at an appropriate time. However, situations may arise when that has not happened and deactivation is required on a more urgent basis.

\subsection{ICDs: method of emergency, temporary deactivation}

Placing a strong magnet on the skin over the ICD generator will suspend tachyarrhythmia detection (and thereby suspend shock delivery) by the device. This can be used to provide emergency, temporary deactivation of its ability to deliver a shock but will not interfere with its ability to function as a pacemaker, where that function is present. Use of a magnet in this way to provide temporary deactivation of defibrillation by an ICD should be regarded as an emergency measure when deactivation as part of end-of-life care is needed without delay, but should not be a usual part of end-of-life care. When a crisis requires emergency use of a magnet in this way as a temporary measure, removal or displacement of the magnet will immediately restore the ability of the ICD to deliver a shock. Therefore, it is important that the magnet is taped securely in position and that repeated checks are made to ensure that it has remained in place.

The ICDs produced by one manufacturer (Biotronik) allow deactivation by a magnet for only $8 \mathrm{~h}$ before the shock function is restored. With this type of ICD (or if the manufacturer is unknown), the magnet should be removed for a few seconds every $7 \mathrm{~h}$ and then taped back into position to ensure continued deactivation. Very rarely, an ICD may have been reprogrammed so that it will not be deactivated by a magnet. In these exceptional circumstances, it is expected that this would have been explained to the patient and documented clearly.

Placing a magnet over a pacemaker that does not have an ICD function will not deactivate it but will cause the pacemaker to fire at a faster than usual rate and will prevent the pacemaker's ability to be inhibited by spontaneous heartbeats. This underlines the importance of knowing exactly the nature of any implanted device and seeking appropriate expert advice before an attempt is made by non-specialists to adjust or inhibit a device.

\subsection{ICDs: explaining deactivation}

When a decision about deactivation of an ICD is being considered, it is especially important that patients and those close to them have a clear understanding of what is being considered, of the reason for and of the expected effect of deactivation. It is common for people to be alarmed by the false belief that deactivation will lead to immediate death, so sensitive, clear and unambiguous explanation is crucial in this situation, as it is in all aspects of end-of-life care. As emphasised elsewhere, employers should ensure that all involved staff receive formal training and that they achieve and maintain competence in undertaking such discussions.

\subsection{ICDs: local device deactivation policy and services}

Local arrangements for ICD deactivation should be recorded clearly as part of the local device management policy. That policy, together with clear instructions on how, where and when to access a magnet, to access help from a cardiac devices physiologist, and to access additional expertise if needed should be readily accessible to all relevant healthcare professionals in all settings, including the community healthcare services, hospitals (especially Emergency Departments, Acute Wards and Assessment Units, Cardiology Wards and Departments) and hospices.

\subsection{ICDs: management of reduced battery life}

As with pacemakers, depletion of its battery to a level that would usually warrant elective replacement of an ICD generator will occur in some people with an ICD who are approaching the end of their life. The decision whether or not to proceed with elective generator replacement in these circumstances must be made on an individual basis after careful assessment of all the circumstances and should usually involve shared decisionmaking with the patient and/or with those close to them, as discussed in section 7. Factors that will be relevant to this decision will include whether or not the device is required for another purpose (ie, pacing for bradycardia or cardiac resynchronisation) and whether or not the patient has reached the stage in their end-of-life care where they have accepted that benefits of receiving defibrillatory shocks from their device no longer exceed the 
potential harms and burdens of generator replacement and continued defibrillator function.

\section{IMPLANTABLE EVENT RECORDERS (ALSO KNOWN AS IMPLANTABLE LOOP RECORDERS OR IMPLANTABLE CARDIAC MONITORS)}

13.1 Implantable event recorders: nature and purpose

Implantable event recorders (IERs) are small devices that can record cardiac rhythm over a prolonged period. They are implanted usually under the skin on the front of the chest, overlying the heart, or occasionally (usually for cosmetic reasons) in the axilla. They monitor the heart's rhythm continuously and will record and store episodes of extreme bradycardia and tachycardia automatically. Also, using a 'remote control device', they can be activated immediately following a symptomatic event (such as transient loss of consciousness) to store the rhythm that was present. They do not deliver any therapy.

\subsection{IERs: removal and non-replacement}

As IERs deliver no therapy, there is no requirement for or ability to deactivate them, or to consider removal if a person with an IER is approaching the end of life. If a person has an IER in place and is then identified as approaching the end of their life, the need for routine attendance for interrogation of the IER should be considered carefully on an individual basis. Removal of an IER is unlikely to be appropriate during end-of-life care as the nuisance and discomfort of the procedure will usually outweigh any possible benefit. The battery life of an IER is usually at least 3 years, and many such devices will have fulfilled their intended purpose within that time so will not require elective replacement. If a person with an IER goes on to develop an advanced illness that brings them towards the end of their life, it is unlikely that elective replacement of an IER that has not already fulfilled its purpose would be appropriate.

\section{IMPLANTABLE NEUROSTIMULATORS}

\subsection{Implantable neurostimulators: nature and purpose}

Neurostimulators are implanted for various indications, detailed discussion of which is beyond the scope of this document. They are similar in size and shape to pacemakers. They are attached to the 'target' part of the nervous system by a lead, similar to a pacemaker lead. The majority are implanted in the wall of the abdomen. However, in some people neurostimulators may be placed subcutaneously in the chest wall, in positions similar to those used for pacemakers. Since they are similar in outward appearance to a pacemaker, this means that they may be mistaken for pacemakers.

\subsection{Implantable neurostimulators-deactivation, removal and non-replacement}

It is unlikely that a deactivation or removal of a neurostimulator would be warranted as part of end-of-life care. Failure of the neurostimulator to deliver the intended treatment could lead to a relapse of the symptoms for which it was implanted. Should neurostimulator battery depletion develop in a person approaching the end of their life, careful consideration should be given to the relative benefits versus the relative harms and burdens of elective device replacement. The situation should be assessed on an individual basis in every person, with full involvement of the experts involved in routine surveillance and management of the device.
Section C: Device management during cardiac arrest

\section{ACTIONS REQUIRED DURING AND AFTER CPR IN PEOPLE WITH IMPLANTED ELECTRONIC DEVICES}

15.1 CPR in people with pacemakers, IERs and neurostimulators

Pacemakers (pacemakers for bradycardia and CRT-P devices), IERs and neurostimulators present no hazard to people providing CPR for cardiorespiratory arrest. No special precautions are necessary when delivering chest compressions and/or ventilation in the presence of any of these devices.

\subsection{Delivery of CPR to a person with an ICD}

In the presence of an ICD (including CRT-D devices), chest compressions and ventilations should be delivered in the recommended, standard way. Although there have been very rare reports of rescuers having felt ICD shocks and experienced transient pain or temporary impairment of nerve conduction, ${ }^{46}$ discharge of a shock from an ICD is believed to present no major risk to another person in contact with the patient as the voltages and current flows recorded from the patient's skin surface are relatively low. ${ }^{47}$ Wearing of 'clinical examination' gloves has been recommended ${ }^{48}$ in the hope that it may reduce to some degree the risk of receiving any shock, even one of low energy. The degree to which gloves may offer such protection to a person delivering CPR is uncertain at the present time. Wearing of gloves is recommended primarily as one of the elements of personal protection against infection for all those delivering CPR.

\subsection{Small risk of lead displacement during CPR}

Healthcare professionals who deliver CPR to a patient with an implanted pacemaker or ICD should be aware that there is a small risk that vigorous chest compressions could result in lead displacement within the heart in some circumstances. The risk of this is very low when the leads have been in place for several months or longer, but is greater if the leads have been implanted relatively recently. However, in the presence of cardiorespiratory arrest, the priority is to provide optimal resuscitation, so the aim should be to deliver good-quality chest compressions, irrespective of the presence of a pacemaker or ICD and irrespective of how recently the leads were implanted.

\subsection{External defibrillation and cardioversion}

Although modern CIEDs are designed to resist damage by external defibrillation or cardioversion currents, there is a remote possibility of damage when a shock is delivered through a defibrillation pad placed over or close to these implanted devices. With pacemakers and ICDs with transvenous/endocardial leads, there is also a theoretical risk of damage to the person's myocardium at the electrode interface due to excess current flow. This may elevate pacing thresholds temporarily or permanently or damage the myocardium temporarily or permanently at the electrode-tissue interface. To minimise this risk, it is recommended that defibrillator electrodes are placed as far away as is practicable from the pacemaker or ICD generator without compromising effective defibrillation. A distance of at least $10-15 \mathrm{~cm}$ between the edge of the device and the edge of the defibrillator electrode is recommended. Placement of the defibrillator electrodes approximately perpendicular to the device and its leads may reduce the risk of current entering the device circuits. If 
necessary, use of alternative electrode positions (eg, anteroposterior) may be used to achieve this. Similar precautions are advised in people with neurostimulators implanted in the chest. In people with IERs, it is advisable to avoid placing defibrillator pads directly over the device to minimise the risk of damage to the device itself, despite in-built protection.

\subsection{ICDs: shockable cardiac arrest rhythms}

An ICD usually gives no warning before it delivers a shock. During an episode of persistent ventricular tachyarrhythmia, an ICD will deliver several shocks before ceasing automatically to give shocks, even if the arrhythmia persists. The precise number of shocks that may be delivered in this situation will vary from one person/device to another, and is often up to eight, sometimes more. The ICD will restart its discharge sequence if it detects even brief apparent cessation of the tachyarrhythmia (including transient slowing of heart rate below the rate programmed to trigger shocks). This could result in the patient receiving a large number of shocks, causing pain and distress.

During cardiorespiratory arrest in a shockable rhythm, external defibrillation should be attempted in the usual way if the ICD has not delivered a shock or if its shocks have failed to terminate the arrhythmia.

\subsection{Use of external pacemakers in the presence of implanted devices}

An external pacemaker may be used for emergency treatment of severe bradycardia and for cardiac arrest in asystole with continued P-wave activity on the ECG.

In the presence of an implanted pacemaker or ICD that has failed and is not emitting any pacing stimuli (seen as 'pacing spikes' on an ECG or monitor), an external pacemaker can be applied and used in the usual way. Electrode position will usually be dictated by the possible need for defibrillation through the same electrodes (see section 15.4).

If the pacemaker or ICD is emitting pacing stimuli but failing to stimulate the heart ('failure to capture'), the pacing spikes from the implanted device may inhibit the external pacemaker. To avoid this, the external pacemaker rate must be faster than the programmed rate of the implanted device and/or the external pacemaker must not be set in 'demand' mode.

If an implanted device is delivering pacing stimuli (at an adequate rate), and each is followed by a QRS complex on the ECG but no detectable cardiac output, that is cardiac arrest with 'pulseless electrical activity'. Use of an external pacemaker will be of no benefit in this situation.

\subsection{Arrange device check and interrogation after successful CPR}

In any patient with an implanted pacemaker or ICD who has ROSC after receiving CPR, an early physiologist's check on the state of the device and its leads should be an integral part of the immediate postresuscitation care to ensure that the device continues to function and deliver treatment appropriately.

Pacemakers and ICDs store information about rhythm behaviour. In the presence of one of these devices, or of an IER, interrogation of the device following ROSC may provide useful information about the rhythm behaviour that initiated the arrest. That information may be an important guide to choice of further treatment.
Section D: Device management after death

\section{ACTIONS REQUIRED AFTER DEATH IN PEOPLE WITH IMPLANTED CARDIOVASCULAR DEVICES}

Local policies should include guidance on how healthcare professionals obtain access to a physiologist to interrogate and/or deactivate a device after death, and the other local arrangements that are in place for device removal, disposal or other management after death. Such policies should make provision for responding appropriately in situations where there is a cultural or religious requirement for early burial or cremation. ${ }^{49}$

\subsection{Immediate actions: pacemakers}

Whether implanted for treatment of bradycardia or for treatment of heart failure, pacemakers usually require no immediate action when someone dies. However, a CRT-D device has an ICD function and must be managed as described in section 16.2 below.

If death occurs suddenly and unexpectedly in someone with a pacemaker, it is important to remember that most pacemakers have a memory function that may provide information about heart rhythm behaviour and device behaviour immediately prior to death, information that may be of help to the Coroner (or in Scotland, the Procurator Fiscal) in identifying the mechanism and cause of death. In these circumstances, a cardiac devices physiologist (usually from the local pacemaker service) should be asked to undertake an early interrogation of the pacemaker, whenever possible prior to its removal, and the detailed findings should be documented in the patient's medical records.

\subsection{Immediate actions: ICDs}

When someone dies with an active ICD (including a CRT-D device) in place, it is important that the device is deactivated as soon as is practicable and certainly before any attempt is made to perform an autopsy or to remove the device. Cutting through the lead to remove an active ICD would place the operator at risk of receiving a shock. There may also be a risk of the device detecting movement or other artefact as a ventricular arrhythmia and delivering a shock that could be transmitted to the person performing the autopsy or device removal. In most expected deaths, it is hoped that end-of-life care planning would have led to deactivation of the device prior to death.

If the death was sudden and not expected at that time, early interrogation of the device by a cardiac devices physiologist should be arranged to seek and document potentially useful information from the ICD, as described in section 16.1 above.

\subsection{Immediate actions: IERs}

If a person dies suddenly or unexpectedly with an IER in place, its early interrogation should be arranged and the findings documented for the same reason.

\subsection{Immediate actions: implantable neurostimulators}

No immediate action is needed after death in people with implanted neurostimulators.

\subsection{Subsequent actions: autopsy, device removal and cremation}

Funeral directors and mortuary attendants who are uncertain about the nature of an implanted device and whether or not it requires deactivation should be encouraged to contact their 
local pacemaker/ICD service in the first instance as they will usually be able to advise and would need to be contacted anyway to deactivate an ICD that remains active after death.

In a dead person with an active ICD, no attempt should be made to undertake an autopsy or remove the device until it has been deactivated, usually by a cardiac physiologist. Temporary deactivation using a magnet is not adequate for these purposes. An ICD that is still active at the time of death should be deactivated as soon as is practicable.

If a person's body is to be cremated, it is important that a pacemaker, ICD (once deactivated), IER or neurostimulator is removed prior to cremation. It is necessary only to remove the pacemaker, ICD or neurostimulator generator; the leads may be left in place. The reason for this is that these generators (and IERs) are sealed units, designed to withstand high pressures. However, heating to a very high temperature is likely to cause the device to explode, creating some resulting hazard and depriving the deceased person and those close to them of a dignified cremation.

The matter of ownership of the implanted device should be considered (see section 7.14) and, where necessary, appropriate consent should be obtained for removal and retention of an implanted device.

\subsection{Subsequent actions: burial}

When burial is intended, there is no absolute need to remove any of these electronic devices. In some instances, removal may be appropriate to allow testing of the device. As in section 16.5 above, where necessary, appropriate consent should be obtained for removal and retention of an implanted device.

\subsection{Disposal of implanted devices after removal}

The following guidance applies equally to

- removal of an implanted electronic device during life for clinical reasons (eg, battery depletion or infection);

- removal of an implanted electronic device after death (eg, for testing of the device or to allow cremation to proceed).

A policy for safe disposal of implantable electronic devices after removal should be followed by every device service.

Devices removed and retained in mortuaries or by funeral directors should be returned to the local device service for safe disposal. The majority of explanted CIEDs are returned to physiologists in hospital device services for safe disposal. All device manufacturers have a disposal policy and supply the necessary means for collection and disposal of devices. Device services should be aware of and should use these arrangements.

In the event of an explanted device being retained by a patient or a beneficiary of a deceased patient, consideration should be given to aspects of health and safety that may apply (including any relevant risk in relation to communicable disease and the risk of explosion if the device is heated). The recipient of the device should be given advice on its safe handling and disposal.

\section{Section E: Policies, quality standards and further reading}

\section{POLICIES GOVERNING DEVICE MANAGEMENT}

Healthcare provider organisations should have a policy for device management that crosses all local organisational boundaries, and that includes clear details of

- the services in that community (and/or at a regional centre if services are not available at all times within the local community) available to support people with implanted cardiac devices and the healthcare professionals caring for those patients, including how to obtain details of any individual patient's device;

- where the policy is a regional one, specific additional information relating to individual provision at local level within that region;

- information leaflets and other resources available to enhance that support and how to access them;

- when to consider device deactivation;

- who is available to advise on decisions about device deactivation and how they should be contacted regarding those decisions;

- how to contact an appropriate cardiac physiologist when deactivation of a device is considered necessary;

- what documentation is required to support or validate a decision to deactivate a device and allow deactivation to proceed without delay;

- how to contact an appropriate cardiologist for advice on device management when necessary;

- how and when to contact palliative care services in support of device deactivation as part of end-of-life care;

- how and where to obtain immediate access to a magnet for emergency, temporary ICD deactivation when necessary and how to apply it;

- information, guidance and support that should accompany the issue of a magnet to a patient with an ICD in those localities where it is standard practice to issue a magnet to each person with an ICD;

- circumstances in which reactivation of a previously deactivated device may be appropriate;

- different actions that are needed during and out of 'office hours';

- different actions that are needed according to the location and condition of the patient;

- specific duties of or actions required from different healthcare professionals in relation to device deactivation;

- local arrangements that are in place for disposal of explanted devices;

- arrangements for training and maintenance of competence of involved staff in undertaking sensitive communication about device management;

- arrangements for training and maintenance of competence of involved staff in carrying out device deactivation;

- actions that should be taken concerning device deactivation, removal or disposal when a person with an implanted cardiac device has died.

In addition, such policies provide an opportunity to promote a clear understanding of the importance of including explanation of the possible later need for device deactivation as part of the process of obtaining properly informed consent, prior to initial device implantation (see section 7.4). They offer an opportunity to provide healthcare professionals with basic understanding of the nature and purpose of implanted devices, and of the balance of benefits and burdens that form the basis of most decisions to deactivate them. This may help to avoid misunderstandings by healthcare professionals and help them to communicate effectively and avoid misunderstandings by patients and those close to them. They offer also an opportunity to provide guidance on the delivery of CPR to people with implanted devices, and appropriate consideration of DNACPR decisions and other decisions relating to end-of-life care.

It is important that such policies are kept up to date and that healthcare staff have prompt access to current policies and 
guidance at all times. In particular, if individual contact names or telephone numbers are included, a mechanism should be in place to update these immediately whenever there is a change of staff or change of contact details. If printed copies of policies are used, they should contain clear warning that they may not be the latest version. Provision of round-the-clock electronic access to the current version of the policy is the recommended approach.

\section{QUALITY STANDARDS FOR DEVICE MANAGEMENT}

All patients with a CIED should have timely access to expert clinical support for their device and should be provided with clear information on how to obtain help whenever they need it. Standards for implantation and follow-up of cardiac rhythm management devices in adults have been defined by the British Heart Rhythm Society (formerly Heart Rhythm UK). ${ }^{50}$

All patients with a CIED should be provided with and encouraged to carry with them information about their device, so that it is available to clinicians in the event of an emergency.

Patients with a CIED should be under regular surveillance in a pacemaker/ICD clinic. The service provided by that clinic should include the provision of information about deactivation of their device should that become necessary or appropriate. The clinic should provide prompt access for patients requiring device deactivation (or reactivation in occasional cases).

The service should provide immediate round-the-clock access to magnets for emergency deactivation of ICDs, and the location of those magnets should be known to all relevant healthcare staff (especially but not exclusively Emergency Department, Acute Medicine, Cardiac Care Unit and Cardiology hospital staff, Palliative Care professionals and Heart Failure Nurse Specialists). In some localities, it is standard practice to issue a magnet to each person with an ICD. Where this is the case, patients and those close to them should also receive information, guidance and ongoing support to ensure that the purpose of the magnet is understood, that the likelihood of appropriate use is optimised and that the likelihood of inappropriate use is minimised.

Arrangements should be in place to provide physiologistdelivered ICD deactivation in another healthcare facility (such as a hospice or nursing home) or in the patient's home, where the patient is sufficiently unwell or close to the end of their life to make travel to a hospital clinic inappropriate.

Arrangements should be in place to provide round-the-clock access to expert cardiological advice to support patients with CIEDs and to support the other healthcare professionals caring for them at any time. If necessary, that may require arrangements for access to advice from a regional centre if the relevant expertise is not available locally at all times.

Arrangements should be in place to provide prompt physiologist-delivered ICD deactivation for any patient who has died with an active ICD in place, to allow safe conduct of an autopsy or safe removal of the device to allow cremation.

Arrangements should be in place to provide prompt physiologist-delivered interrogation of pacemakers, IERs or ICDs when a patient with one of these devices dies suddenly and unexpectedly. Those responsible for investigating the cause of such deaths (eg, Coroners' Pathologists, Medical Examiners) should be aware of these arrangements and of the potential information that may be obtained in this way.

\section{Glossary of abbreviations}

Each abbreviation is defined at least once in the text, but this glossary is provided also for ease of reference.

- ADRT Advance decision to refuse treatment

- AV Atrioventricular

- CIED Cardiovascular implantable electronic device

- CPR Cardiopulmonary resuscitation

- CRT Cardiac resynchronisation therapy (biventricular pacing)

- CRT-D Cardiac resynchronisation therapy (biventricular pacing) that also has an ICD function

- CRT-P Cardiac resynchronisation therapy (biventricular pacing) with no ICD function

- DNACPR Do not attempt cardiopulmonary resuscitation

- ECG Electrocardiogram

- ICD Implantable cardioverter defibrillator

- IER Implantable event recorder (known also as an implantable loop recorder or implantable cardiac monitor)

- ROSC Return of spontaneous circulation

- VF Ventricular fibrillation

- VT Ventricular tachycardia

\section{Author affiliations}

${ }^{1}$ Vice President, Resuscitation Council (UK), 5th Floor, Tavistock House North, Tavistock Square, London, WC1H 9HR, UK

${ }^{2}$ Consultant in Anaesthetics \& Intensive Care Medicine, North Bristol NHS Trust, Bristol, UK

${ }^{3}$ Consultant Cardiologist, Glasgow Royal Infirmary, Glasgow, UK

${ }^{4}$ Consultant Cardiologist, James Cook University Hospital, Middlesbrough, UK ${ }^{5}$ Director of Policy \& External Affairs, the National Council for Palliative Care, London, UK

${ }^{6}$ Consultant Cardiologist, Heart of England NHS Foundation Trust, Birmingham, UK ${ }^{7}$ Pacing/ICD Service Manager, St George's Healthcare NHS Trust, London, UK

${ }^{8}$ Medical Director, St Christopher's Hospice, Consultant Physician in Palliative Care,

Guy's \& St Thomas' NHS Foundation Trust, Professor of Palliative Care, Cicely

Saunders Institute, King's College London, London, UK

${ }^{9}$ Consultant Cardiologist, Freeman Hospital, Newcastle upon Tyne, UK

${ }^{10}$ Consultant Cardiologist, County Hospital, Hereford, UK

${ }^{11}$ Member of the Patient Advisory Group, Resuscitation Council (UK), London, UK

${ }^{12}$ Lead Resuscitation Officer, Papworth Hospital, Cambridge, UK

${ }^{13}$ Director of Courses Development and Training, Resuscitation Council (UK), London, UK

${ }^{14}$ Consultant in Emergency Medicine, Calderdale Royal Hospital, Halifax, UK

${ }^{15}$ Transplant \& Divisional Support Manager, Heart Division, Harefield Hospital, Harefield, UK

${ }^{16}$ Executive Director, Resuscitation Council (UK), London, UK

Acknowledgements The Working Group is grateful to: Dr Graham Stuart, Consultant Cardiologist (Congenital Heart Disease), Bristol Royal Hospital for Children for reviewing the document from a paediatric perspective and contributing to the wording of 'People (patients) considered in this document'. Professor John Coggon, Centre for Health, Ethics and Law, Southampton Law School, University of Southampton and Professor Richard Huxtable, Professor of Medical Ethics and Law, Deputy Director, Centre for Ethics in Medicine, School of Social and Community Medicine, University of Bristol for reviewing and editing the document from a legal perspective. Professor Margaret Brazier, Professor of Law, School of Law, University of Manchester and Dr Muireann Quigley, Senior Lecturer in Biomedical Ethics and Law, Centre for Ethics in Medicine, University of Bristol for advice regarding ownership of implanted devices.

Contributors DP chaired the Working Group, and compiled the initial draft of the guidance. JS and DP carried out the literature review. All authors contributed to the development of the guidance by expressing their views and those of their groups, organisations or specialties at meetings of the Working Group and/or in email communications between meetings. All authors contributed to further development of the text of the guidance and agreed the final wording. 
Competing interests None declared.

Provenance and peer review Commissioned; internally peer reviewed.

\section{REFERENCES}

1 Colquhoun $M$, Jones $S$, Clarke $T$, et al. Emergency management of arrhythmias and/ or shocks in patients with implantable cardioverter defibrillators (ICDs). A statement on behalf of the Resuscitation Council (UK), Heart Rhythm UK (formerly The British Pacing and Electrophysiology Group, BPEG), The Joint Royal Colleges Ambulance Liaison Committee (JRCALC) and the Ambulance Services Association (ASA). Resuscitation 2006;71:278-82.

2 Padeletti L, Arnar DO, Boncinelli L, et al. EHRA Expert Consensus Statement on the management of cardiovascular implantable electronic devices in patients nearing end of life or requesting withdrawal of therapy. Europace 2010;12:1480-9.

3 Lampert R, Hayes DL, Annas GJ, et al. HRS Expert Consensus Statement on the Management of Cardiovascular Implantable Electronic Devices (CIEDs) in patients nearing end of life or requesting withdrawal of therapy. Heart Rhythm 2010;7:1008-26.

4 Pettit SJ, Browne S, Hogg KJ, et al. ICDs in end-stage heart failure. BMJ Support Palliat Care 2012:2:94-7.

5 Resuscitation Council (UK). Resuscitation Guidelines 2015. https://www.resus.org. uk/resuscitation-guidelines/ (accessed Apr 2016).

6 General Medical Council 2007. 0-18 years: guidance for doctors. http://www. gmc-uk.org/guidance/ethical_guidance/children_guidance_22_23_making_ decisions.asp (accessed Apr 2016).

7 Larcher V, Craig F, Bhogal K, et al. on behalf of the Royal College of Paediatrics and Child Health. Making decisions to limit treatment in life-limiting and life-threatening conditions in children: a framework for practice. Arch Dis Child 2015;100:s1-23. http://adc.bmj.com/content/100/Suppl_2/s1.full (accessed Apr 2016)

8 UK Government. Family Law Reform Act 1969. http://www.legislation.gov.uk/ukpga/ 1969/46/section/8 (accessed Apr 2016).

9 Kinch Westerdahl A, Sjöblom J, Mattiasson AC, et al. Implantable cardioverter-defibrillator therapy before death: high risk for painful shocks at end of life. Circulation 2014;129:422-9.

10 Carter C. Dying grandfather 'shocked back to life' 30 times. http://www.telegraph. co.uk/health/healthnews/10991824/Dying-grandfather-shocked-back-tolife-30-times.html (accessed Apr 2016).

11 National Institute for Health and Care Excellence 2010. Chronic heart failure: management of chronic heart failure in adults in primary and secondary care. http:/l www.nice.org.uk/guidance/CG108 (accessed Apr 2016).

12 National Institute for Health and Care Excellence 2011. QS9 Chronic heart failure quality standard. http://www.nice.org.uk/guidance/qs9 (accessed Apr 2016).

13 Dickstein K, Vardas PE, Auricchio A, et al. 2010 Focused Update of ESC Guidelines on device therapy in heart failure: an update of the 2008 ESC Guidelines for the diagnosis and treatment of acute and chronic heart failure and the 2007 ESC guidelines for cardiac and resynchronization therapy. Developed with the special contribution of the Heart Failure Association and the European Heart Rhythm Association. Eur Heart J 2010;31:2677-87.

14 Dying Matters Coalition. Identifying end of life patients. http://dyingmatters.org/gp_ page/identifying-end-life-patients (accessed Apr 2016).

15 The National Gold Standards Framework Centre in End of Life Care. http://www. goldstandardsframework.org.uk/home (accessed Apr 2016).

16 Royal College of General Practitioners. End of life care resources for GPs. http:// www.rcgp.org.uk/endoflifecare (accessed Apr 2016).

17 Stone ME, Salter B, Fischer A. Perioperative management of patients with cardiac implantable electronic devices. Br J Anaesth 2011;107(Suppl 1):i16-26.

18 Brignole M, Auricchio A, Baron-Esquivias G, et al. 2013 ESC Guidelines on cardiac pacing and cardiac resynchronization therapy: the Task Force on cardiac pacing and resynchronization therapy of the European Society of Cardiology (ESC). Developed in Collaboration with the European Heart Rhythm Association. Eur Heart $J$ 2013;34:2281-329.

19 General Medical Council 2008. Consent: patients and doctors making decisions together. http://www.gmc-uk.org/publications/standards_guidance_for_doctors.asp (accessed Apr 2016).

20 General Medical Council 2010. Treatment and care towards the end of life: good practice in decision making. http://www.gmc-uk.org/publications/standards_ guidance_for_doctors.asp (accessed Apr 2016).

21 England R, England T, Coggon J. The ethical and legal implications of deactivating an implantable cardioverter-defibrillator in a patient with terminal cancer. J Med Ethics 2007:33:538-40.

22 Wu EB. The ethics of implantable devices. J Med Ethics 2007;33:532-3.

23 English V. Withholding and withdrawing life-prolonging medical treatment. 3rd edn. London: BMJ Books, 2007
24 Pedersen SS, Chaitsing R, Szili-Torok T, et al. Patients' perspective on deactivation of the implantable cardioverter-defibrillator near the end of life. Am J Cardiol 2013;111:1443-7.

25 Fluur C, Bolse K, Strömberg A, et al. Patients' experiences of the implantable cardioverter defibrillator (ICD); with a focus on battery replacement and end-of-life issues. Heart Lung 2013:42:202-7.

26 Goldstein N, Bradley E, Zeidman J, et al. Barriers to conversations about deactivation of implantable defibrillators in seriously ill patients: results of a nationwide survey comparing cardiology specialists to primary care physicians. J Am Coll Cardiol 2009;54:371-3.

27 Marinskis $G$, van Erven $L$, on behalf of the EHRA Scientific Initiatives Committee. Deactivation of implanted cardioverterdefibrillators at the end of life: results of the EHRA survey. Europace 2010;12:1176-7.

28 Raphael C, Kanagaratnam P, Francis DP. Further barriers to conversations about deactivation of implantable cardioverter-defibrillators. J Am Coll Cardiol 2010;55:701-2.

29 Clark AM, Jaarsma T, Strachan $\mathrm{P}$, et al. Effective communication and ethical consent in decisions related to ICDs. Nat Rev Cardiol 2011;8:1-12.

30 Niewald A, Broxterman J, Rosell T, et al. Documented consent process for implantable cardioverter-defibrillators and implications for end-of-life care in older adults. J Med Ethics 2013:39:94-7.

31 Carroll SL, Strachan PH, de Laat S, et al. Patients' decision making to accept or decline an implantable cardioverter defibrillator for primary prevention of sudden cardiac death. Health Expect 2013;16:69-79.

32 Ågård A, Löfmark R, Edvardsson N, et al. Views of patients with heart failure about their role in the decision to start implantable cardioverter defibrillator treatment: prescription rather than participation. J Med Ethics 2007;33:514-18.

33 H M Government. Mental Capacity Act 2005. London: The Stationery Office, 2005. http://www.legislation.gov.uk/ukpga/2005/9/contents (accessed Apr 2016).

34 Department for Constitutional Affairs. Mental Capacity Act 2005: code of practice. London: The Stationery Office, 2007. https://www.gov.uk/government/publications/ mental-capacity-act-code-of-practice (accessed Apr 2016).

35 H M Government. Adults with Incapacity (Scotland) Act 2000. The Stationery Office, 2000. http://www.legislation.gov.uk/asp/2000/4/contents (accessed Apr 2016).

36 The Scottish Government. Adults with Incapacity (Scotland) Act 2000: A short guide to the Act. http://www.scotland.gov.uk/Publications/2008/03/25120154/1 (accessed Apr 2016).

37 UK Government. Make, register or end a lasting power of attorney. https://www. gov.uk/power-of-attorney/overview (accessed Apr 2016).

38 NHS National End of Life Care Programme. Capacity, Care Planning And Advance Care Planning In Life Limiting IIIness. A Guide for Health and Social Care Staff. http://www. ncpc.org.uk/sites/default/files/ACP_Booklet_June_2011.pdf (accessed Apr 2016).

39 Kramer DB, Buxton AE, Zimetbaum PJ. Time for a change-a new approach to ICD replacement. N Engl J Med 2012;366:291-3.

40 British Medical Association, Resuscitation Council (UK) and Royal College of Nursing 2014. Decisions relating to cardiopulmonary resuscitation. http://www.resus.org.uk/ pages/dnacpr.htm (accessed Apr 2016).

41 Department of Health and Social Security. Health services management ownership of implants and removal of cardiac pacemakers after death. London: Health Notice HN (83)6, 1983.

42 Beattie J. ICD deactivation at the end of life: Principles and practice. A discussion document for healthcare professionals. London: British Heart Foundation, 2013.

43 McMurray JJV, Adamopoulos S, Anker SD, et al. ESC Guidelines for the diagnosis and treatment of acute and chronic heart failure 2012: the Task Force for the Diagnosis and Treatment of Acute and Chronic Heart Failure 2012 of the European Society of Cardiology. Developed in collaboration with the Heart Failure Association (HFA) of the ESC. Eur Heart J 2012:33:1787-847.

44 Singh JP, Gras D. Biventricular pacing: current trends and future strategies. Eur Heart J 2012;33:305-13.

45 Cappato R, Smith WM, Hood MA, et al. Subcutaneous chronic implantable defibrillation systems in humans. J Interv Card Electrophysiol 2012;34:325-32.

46 Stockwell B, Bellis G, Mortona G, et al. Electrical injury during "hands on" defibrillation-A potential risk of internal cardioverter defibrillators? Resuscitation 2009:80:832-4.

47 Peters $W$, Kowallik $P$, Reisberg $M$, et al. Body surface potentials during discharge of the implantable cardioverter defibrillator. J Cardiovasc Electrophysiol 1998;9:491-7.

48 Nolan JP, Soar J, Zideman DA, et al. European Resuscitation Council Guidelines for Resuscitation 2010 Section 1. Executive summary. Resuscitation 2010;81:1219-76.

49 Wilson J, on behalf of National Nurse Consultant Group (Palliative Care). Care After Death: Guidance for staff responsible for care after death (second edition). http:/l www.hospiceuk.org/what-we-offer/publications?cat=72e54312-4ccd-608d-ad24ff0000fd3330 (accessed Apr 2016).

50 Heart Rhythm UK. Standards for implantation and follow-up of cardiac rhythm management devices in adults. January 2013. http://www.bhrs.com/standards (accessed Apr 2016) 